\title{
Unique Features of the Mode of Action of ET-743
}

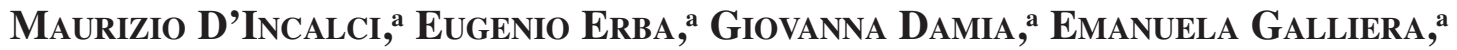 \\ Laura Carrassa, ${ }^{\mathrm{a}}$ Sergio Marchini, ${ }^{\mathrm{a}}$ Roberto Mantovani, ${ }^{\mathrm{b}}$ Gianluca Tognon, ${ }^{\mathrm{a}}$ \\ Robert Fruscio, ${ }^{a}$ José Jimeno, ${ }^{c}$ Glynn T. Faircloth ${ }^{d}$
}

aDepartment of Oncology, Istituto di Ricerche Farmacologiche "Mario Negri," Milan, Italy; 'Dipartimento di Biologia Animale, Università degli Studi di Modena e Reggio Emilia, Modena, Italy; ${ }^{\circ}$ PharmaMar SA, Research and Development, Madrid, Spain; ${ }^{~}$ PharmaMar USA, Inc., Cambridge Massachusetts, USA

Key Words. ET-743 · Natural product $\cdot$ Marine compound $\cdot$ Anticancer drug

\begin{abstract}
This paper describes the current knowledge of the primary mode of action of a natural product, ecteinascidin 743 (ET-743), derived from the marine tunicate Ecteinascidia turbinata. ET-743 was initially selected for preclinical development because of its potent antitumor activity observed against several human solid tumor types. In vitro, the drug is cytotoxic in the nanomolar range, and in the case of some very sensitive cell lines, in the picomolar range. The large potency differences observed among several solid tumor types indicate that this compound possesses some tumor selectivity, but the molecular basis of these differential effects remains to be elucidated. The present studies

were undertaken to evaluate the mechanism of action of ET-743 in this context. The available information on ET-743 binding to DNA and its effects on transcriptional regulation point to a unique behavior of this drug, as it independently affects specific gene transcription in a promoter-dependent way. In addition, ET-743 shows a peculiar pattern of selectivity in cells with different defects in their DNA-repair pathways. These results highlight a unique property of ET-743, possibly explaining why it possesses antitumor activity against tumors that are refractory to standard anticancer drugs, all of which certainly act by mechanisms that are different from that of ET-743. The Oncologist 2002;7:210-216
\end{abstract}

\section{INTRODUCTION}

Although several alterations of mechanisms controlling proliferation, differentiation, and cell death have been identified in many human neoplasms, we are still far from understanding the key reactions to be blocked to cure cancer. Specific therapies able to hit relevant targets can be realistically developed for only a limited number of neoplastic diseases. For this reason, it seems reasonable to explore whether novel natural products discovered by empirical approaches are effective against a variety of human cancers.

This paper focuses on a natural product of marine origin, ecteinascidin 743 (ET-743) (Fig. 1), isolated from the

Figure 1. Molecular structure of ecteinascidin 743 (ET-743).

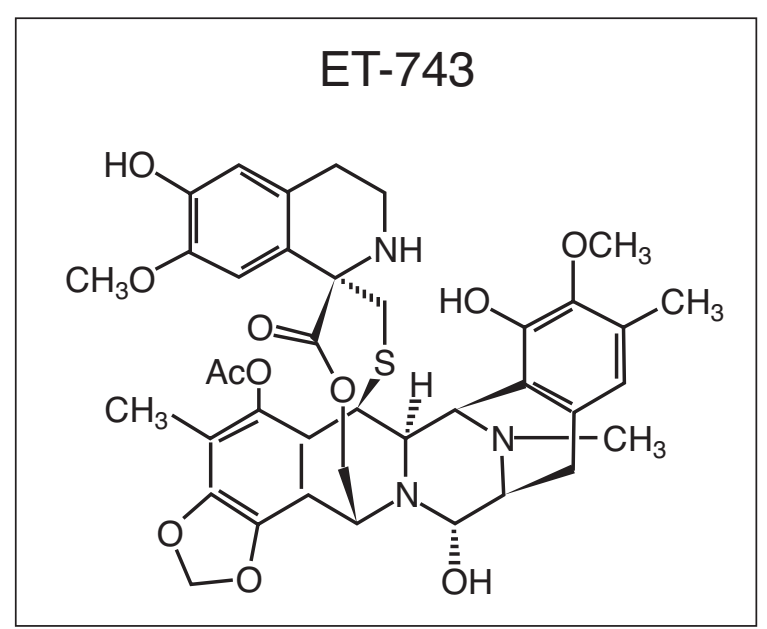

Correspondence: Maurizio D'Incalci, M.D., Department of Oncology, Istituto di Ricerche Farmacologiche "Mario Negri”, Via Eritrea 62, 20157 Milan, Italy. Telephone: 39-02-3901-4478; Fax: 39-02-354-6277; e-mail: dincalci@marionegri.it Received August 13, 2001; accepted for publication March 18, 2002. C)AlphaMed Press 1083-7159/2002/\$5.00/0 
Caribbean tunicate Ecteinascidia turbinata, as an example of an effective novel drug [1-5] that can be considered the prototype of a new class of anticancer compounds. ET-743 is currently in phase II clinical trials and has shown evidence of activity in soft tissue sarcomas and in breast and ovarian cancers [6-9]. The main body of information on the mode of action of this drug is critically reviewed, particularly in relation to the studies recently performed by our laboratories.

\section{BINDING TO DNA}

Studies performed by Pommier et al. [10] at the National Cancer Institute have demonstrated that ET-743 forms DNA adducts. These authors incubated, in vitro, radiolabeled DNA fragments with ET-743, evaluated the shift of electrophoretic migration, and analyzed the sequence-specific binding by footprinting techniques. These studies indicated that the ET-743-DNA adducts involved the exocyclic $\mathrm{N} 2$ amino group of guanine located in the DNA minor groove. This type of alkylation depends on dehydration of the carbinolamine functional group of ET-743 leading to the formation of an iminium intermediate which subsequently reacts with and causes binding to DNA.

Another important contribution to the understanding of how ET-743 binds to DNA was made by Hurley and coworkers. They reported a nuclear magnetic resonance (NMR)-based model of ET-743 binding to duplex DNA indicating that the A and B subunits of the drug are responsible for DNA recognition and bonding, whereas the $\mathrm{C}$ subunit does not have contact with DNA and protrudes out from the minor groove $[11,12]$. Gel shift assays combined with high-field NMR have demonstrated that the alkylation of ET-743 occurs at guanines located either in the sequence $5^{\prime}$ PuGC-3' or $5^{\prime}$-PyrGG-3' [10-13]. Other adducts are formed, but are less stable and are reversible [12-14], suggesting that, after prolonged exposure, ET-743 would be released from these less stable adducts and a progressively higher percentage of ET-743 would be found bound to guanines located in the frame of bases conferring high thermodynamic stability. The reported structural changes induced by ET-743, such as widening of the minor groove and bending of DNA toward its major groove, could be important in affecting the recognition and binding of transcription factors or DNA-binding proteins (e.g., DNA repair proteins). The changes in bending of DNA induced by ET-743 could be, in some cases, incompatible with the binding of some transcriptional factors, whereas, in other cases, bending could be enhanced. The precise characterization of these effects might explain why ET-743 simultaneously downregulates some genes and upregulates others (see next section).

\section{IN VITRO INTERFERENCE OF ET-743 WITH DNA-Binding Proteins and Transcriptional REgULATION}

The main structural differences between ET-743 and the antibiotic saframycin, which does not possess antitumor properties, is a tetrahydroisoquinolone $\mathrm{C}$ subunit, which is absent in saframycin A. This observation prompted us to speculate that this part of the molecule is crucial for the mechanisms responsible for the antitumor activity. According to the model proposed by Hurley and coworkers $[11,12,14]$, it seems that a $C$ subunit not bound to DNA but projecting out from the minor groove might be able to interfere with DNA-binding factors. This hypothesis led us to investigate whether transcription factors that bind to DNA were inhibited by ET-743, which also binds to DNA [15]. We selected three types of factors: A) oncogene products, such as MYC, c-MYB, and Maf; B) transcriptional activators regulated during the cell cycle, such as E2F and SRF, and C) general transcription factors, such as Sp1, TATA-binding protein (TBP), and NF-Y. No inhibition of DNA binding was found for Sp1, Maf, MYB, or MYC, while for TBP, E2F, and SRF, inhibition was observed at concentrations higher than $50 \mu \mathrm{M}$. The inhibition of NF-Y binding was found to occur at ET-743 concentrations in the range of 10-30 $\mu \mathrm{M}$. Since two subunits of NF-Y have a high analogy with histone 2B, the effect of ET-743 on nucleosome reconstitution (i.e., a chromatine-like fiber formed in vitro by adding histones to DNA) was investigated, and it was found that a complete inhibition occurred at drug concentrations of 3-10 $\mu \mathrm{M}$. These data would suggest that the mode of action correlates with the ability of ET-743 to interfere with the binding of regulatory proteins to DNA, thus altering their specific function. However, we have reported these data with caution since the observed effect occurs at ET-743 concentrations that are higher than $1 \mu \mathrm{M}$; whereas, the in vitro cytotoxic activity of ET-743 consistently occurs in a much lower range of 10 picomolar to 10 nanomolar, at least two logs lower than the concentrations used for the DNA binding studies.

Since these in vitro data indicated that one of the factors that was preferentially affected by ET-743 was NF-Y, we focused our attention on this factor, because NF-Y activates the CCAAT element present in $25 \%$ of eukaryotic promoters, including many promoters that regulate genes controlling the cell cycle.

NIH-3T3 fibroblasts transfected with a reporter gene under the control of HSP70 promoter, contain two CCAAT boxes activated by NF-Y. In this system, it is possible to demonstrate that a short treatment with ET-743 in the nM range inhibits the induction of transcription of HSP70 [16]. This promoter-dependent effect was also seen by others for the MDR1 gene [17], which contains NF-Y binding sites. 
A recent study indicates that several genes are either down- or upregulated [18] in intestinal carcinoma HCT116 and breast MDA-MB-435 cell lines exposed to ET-743. It is important to realize that not all genes are transcriptionally regulated by NF-Y, thus indicating that other mechanisms must be involved in the transcriptional effects caused by ET-743. The authors of this report have also found that a synthetic ana$\log$ of ET-743, phthalascidin (Pt650), causes similar effects on gene expression to those caused by ET-743 [18].

Synold et al. [19] recently reported that the orphan nuclear receptor, SXR, coordinately regulates drug metabolism through the induction of transcription of the gene encoding for the cytochrome P450 enzyme, CYP3A4, as well as the transcription of MDR1, a gene encoding Pgp, a glycoprotein that regulates the efflux of several compounds including several anticancer drugs. They found that many xenobiotics, including drugs such as taxol, activate SXR, thus enhancing both drug metabolism and its Pgp-mediated drug clearance. ET-743 was found to be able to inhibit SXR and consequently suppressed MDR1 gene transcription. The inhibition of SXR, which appears to have several detoxification genes as targets, might be relevant in relation to the toxicity of ET-743 and of other drugs combined with ET743. The interesting paper by Synold et al. [19] has shown a mechanism occurring in vitro. Further studies should be performed to evaluate the relevance of SXR inhibition in wellcharacterized cellular systems and in vivo to evaluate whether therapeutic doses of ET-743 correlate with the inhibition of SXR and to investigate any implications to the pharmacological and toxicological properties of ET-743.

Takebayashi et al. reported that topoisomerase I was the target of ET-743 [20] resulting in the formation of DNA breaks. This evidence is not very convincing, as the experiments were performed by treating tumor cells with $10 \mu \mathrm{M}$ ET-743, a concentration 500- to 1,000-fold higher than optimal $50 \%$ inhibitory concentration values. As pointed out by Martinez at al. [21], who synthesized ET-743 and other related molecules, topoisomerase I cannot be the primary target, considering that DNA topoisomerase I cross-links are demonstrated only at concentrations several logs greater than the cytotoxic ones. More recently, we provided more definitive evidence that topoisomerase I cannot be the primary target of ET-743, since the drug is equally active in wild-type yeast and in yeast with a deletion in the DNA topoisomerase I gene [22]. The same researchers who suggested that topoisomerase I was the target of ET-743, when performing comparative experiments on camptothecinresistant mouse leukemia P388/CPT45 cells and on the parent p388 cells, realized that they have similar sensitivity, and thus, proposed that the cytotoxicity was independent of topoisomerase I expression [23].
Table 1. Cytotoxicity of DDP and ET-743 in NER proficient and NER deficient cell lines

\begin{tabular}{lccc} 
& AA8 & UV96 & ERA5 \\
DDP $(\mu \mathrm{M})$ & 27.2 & 0.47 & 11.6 \\
ET-743 $(\mathrm{nM})$ & 0.59 & $>4$ & 0.9 \\
\hline
\end{tabular}

Shown are the $50 \%$ inhibitory concentrations of cisplatin (DDP) and ET-743 in a Chinese hamster ovary parental cell line (AA8), an ERCC1-deficient cell line (UV96), and a UV96 ERCC1-complemented cell line (ERA5). Values are the mean of two experiments done in quintuplicate. Standard error is not shown, being less than $10 \%$.

\section{REPAIR MECHANISMS OF ET-743-INDUCED DNA DAMAGE}

To our knowledge, no data are available on the repair of ET-743-induced DNA lesions in mammalian cells. To shed some light on this point, we have investigated ET-743 cytotoxicity in cell lines with specific defects in DNA repair mechanisms: mismatch repair, nucleotide excision repair, and DNA-dependent protein kinase activity [22, 24]. Cell lines with functional impairment of mismatch repair (MMR), which are resistant to cisplatin, were as sensitive to ET-743 as cell lines that are MMR proficient. ET-743 was found to be about fivefold more active in cells lacking the catalytic subunit of DNA-dependent protein kinase (DNAPK) and treatment with wortmannin, a potent inhibitor of DNA-PK [22], sensitized the cells to ET-743.

A very unique response was observed in cells deficient in nucleotide excision repair (NER). ET-743 did show decreased cytotoxic activity in NER-deficient cell lines compared with NER-proficient cell lines [22, 25]. Although the precise mechanism by which NER-deficient cells become less sensitive is not well understood, these findings further highlight the peculiar and unique interaction between ET-743 and DNA. To our knowledge, all known DNA-interacting drugs are, in fact, either more effective or equally effective in NER-deficient cells as in NER-proficient cells. As an example, in Table 1, ET-743 and cisplatin exhibited opposing patterns of sensitivity in ERCC1-deficient cells and in transfected cells in which the human ERCC1 gene had been inserted. Recently these findings were confirmed by other laboratories in different systems [13, 25]. Table 2 highlights the different patterns of sensitivity to ET-743, cisplatin, and UV light in cells with defined DNA repair defects.

An interesting study, recently published by Zewail-Foote et al. [13], showed that not all ET-743-DNA adducts are equally incised by Uvr ABC. The ET-743 adducts at the nonpreferred sequences (e.g. 5'-AGC and 5'-TGC) (see section on DNA binding) were incised with the highest efficiency, whereas adducts at the preferred sequences $\left(5^{\prime}\right.$-AGC and 


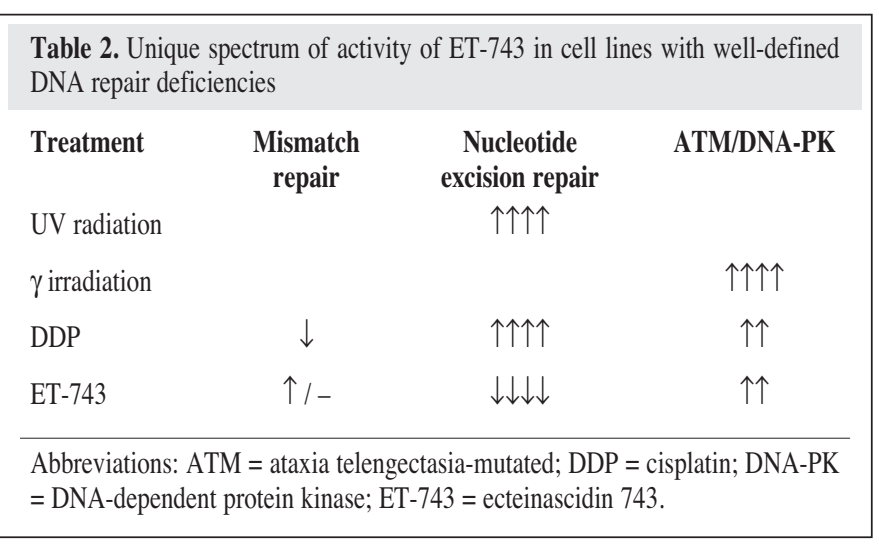

$5^{\prime}$-TGC) were incised to a lesser extent. These authors proposed that the adducts that are not efficiently repaired trap DNA-nucleotide excision repair proteins forming cytotoxic complexes. This interesting hypothesis, if verified, could have important implications for use with other DNAdamaging agents in combinations with ET-743, including radiation.

\section{Cell Cycle Perturbations and Phase SPECIFICITY}

The effects of 1 hour of ET-743 exposure, evaluated by a BrdUrd/DNA biparametric flow-cytometric analysis, showed that cells in the $\mathrm{S}$ phase during drug treatment progressed through this phase of the cell cycle more slowly than control cells [26]. Also, cells that were in the $G_{1}$ phase progressed through the $\mathrm{S}$ phase slowly. At 24 hours, a high percentage of ET-743-treated cells were arrested in the $\mathrm{G}_{2}$ phase, and only 120 or 168 hours afterwards, depending on the cell line, progressed to mitosis and restarted a new cell cycle.

By synchronizing colon cancer SW620 cells by elutriation, the sensitivity to ET-743 was comparatively evaluated in cells in $G_{1} S$, and $G_{2}-M$ phases. The results showed that the highest sensitivity to the drug occurred when cells were in $G_{1}$, and the lowest occurred when cells were in $G_{2}-M$ phase. This finding is of interest, as the majority of available anticancer drugs that interact with DNA are preferentially effective in S-phase cells, thus indicating a biological effect of ET-743 that is distinct from those observed for the other drugs.

Erba et al. [26] showed that ET-743 induces a significant increase in p53 levels, which promotes apoptosis in cell lines expressing wild-type p53. However, p53 status does not appear to be relevant to the sensitivity to ET-743, as cytotoxic activity does not correlate with the p53 status of different cell lines [24, 26], and the cytotoxicity is not significantly different in p53 (-/-) or (+/+) mouse embryo fibroblasts or in A2780 ovarian cancer cells or cells from the
A2780/CX3 subline transfected with a dominant-negative mutant TP53 [26].

Unpublished data by this laboratory (Vikhanskaya, unpublished observation) indicate that isogenic cell lines expressing different levels of p73 exhibit a similar sensitivity to ET-743, although a decrease in the sensitivity to cisplatin was found in cell lines overexpressing p73 [27].

\section{Combination of ET-743 with Other Drugs}

Considering the profound mechanistic differences between ET-743 and other anticancer drugs, it was logical to try to evaluate ET-743-based combinations. Preclinical data indicate a synergy between ET-743 and anthracyclines, taxanes, and cisplatin. What is of particular interest is the potentiation of activity of antitumor drugs with ET743 concentrations that alone produced moderate or no antitumor effects. An example of this observation is shown in Figure 2. ET-743 alone, at the concentration of $0.25 \mathrm{nM}$ for 24 hours, did not cause any detectable growth inhibition, but enhanced significantly the cytotoxicity of cisplatin. This finding means that ET-743 may be used at lower, relatively nontoxic, doses to potentiate the antitumor activity of cisplatin. Ongoing in vivo studies in xenografts appear to confirm that cisplatin efficacy can be increased dramatically by the concomitant use of ET-743, even at doses that alone produce no apparent effect [28].

Sequence-dependent synergies have also been observed when combining ET-743 with doxorubicin or with paclitaxel in soft tissue sarcoma cell lines [29]. The most favorable effects were seen by treating the cells with ET-743 first followed by doxorubicin, and by exposing the cells to paclitaxel followed by ET-743.

\section{Clinical Investigation}

The phase I program with ET-743 in adult patients has included six phase I trials investigating different schedules [7, 9, 30-32]. Dose-limiting toxicities were hematological toxicities and rhabdomyolysis. At the recommended dose, reversible and noncumulative transaminitis was noted in most of the patients. Evidence of objective activity, including long-lasting responses, was noted in melanoma, breast cancer, ovarian cancer, mesothelioma, and sarcoma. Among 20 patients with advanced pretreated sarcoma entered into different phase I trials at the recommended dose or at the maximal tolerated dose, one had a complete response, three had partial responses, and two had minor responses, establishing a solid foundation to further investigate the activity of ET-743 in connective tissue tumors.

ET-743 is under active phase II/III development in different tumor types. The phase II data available suggest 
Figure 2. Cytotoxic effect of 24hour exposure with ecteinascidin 743 (ET-743) alone, cisplatin (DDP) alone, or ET-743 and DDP in combination evaluated after 7 days by sulforhodamine $B$ test. Mean values of 7-10 replicates and standard error (SE) are reported. When SE is not reported, it was less than $1 \%$ of the mean value.ET-743 at the concentration of $0.25 \mathrm{nM}$ produced no detectable inhibition of growth, whereas it potentiated the cytotoxicity of DDP in a highly significant manner $(\mathrm{p}<0.01)$.

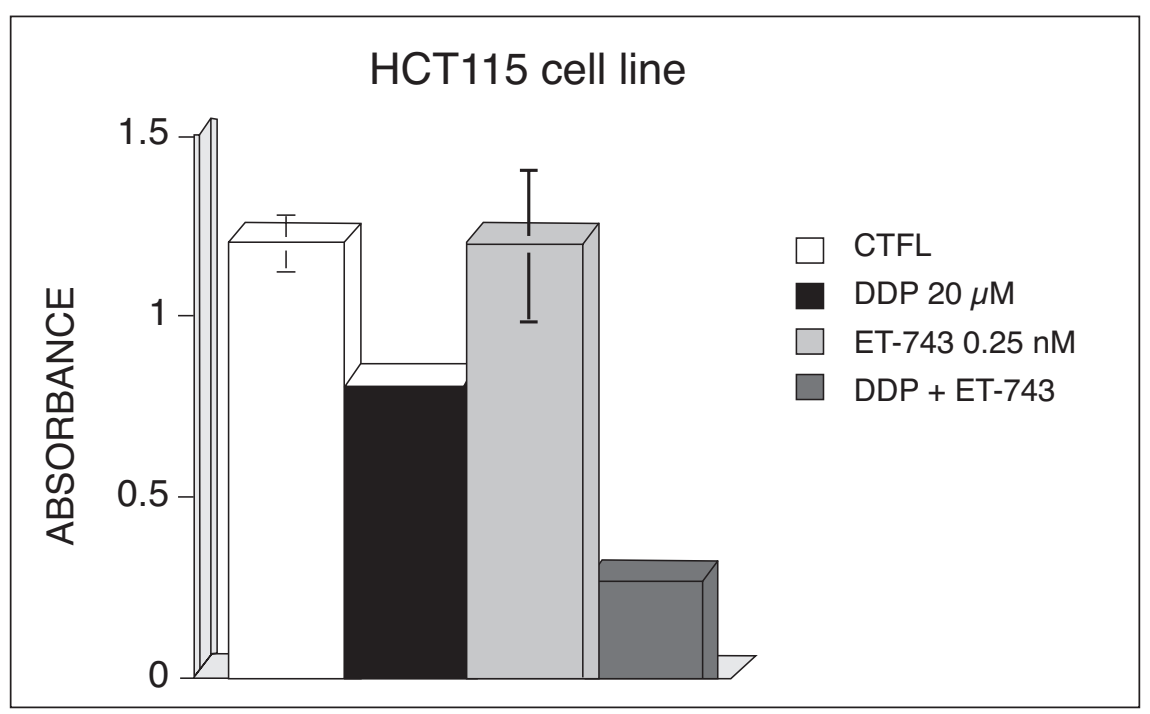

Table 3. Summary of phase II data with ET-743 in advanced soft tissue sarcoma (STS)

$\begin{array}{lclccccc}\text { Investigators } & \text { Tumor type } & \text { Setting } & \boldsymbol{n} \text { of patients } & \text { CR } & \text { PR } & \text { MR } & \text { Median survival } \\ \text { Demetri et al. [34] } & \text { STS* } & 1 \text { line } & 34 & 2 & 3 & 3 & 19 \text { months } \\ \text { Demetri et al. [34] } & \text { STS } & 2 / 3 \text { line } & 36 & 1 & 2 & 2 & 12.1 \text { months } \\ \text { Yovine et al. [33] } & \text { STS } & 2 / 3>3 & 54 & - & 2 & 2 & 13 \text { months } \\ \text { Le Cesne et al. [35] } & \text { STS } & 2 / 3 \text { line } & 99 & - & 9 & 7 & 9.11 \text { months }\end{array}$

$1.5 \mathrm{mg} / \mathrm{m}^{2}$ i.v. 24-hour infusion on day 1 every $3-4$ weeks.

Abbreviations: ET-743 = ecteinascidin 743; CR = complete response; $P R=$ partial response; MR = minor response.

\begin{tabular}{|c|c|c|c|c|c|c|c|}
\hline Investigators & Tumor type & Setting & $n$ of patients & CR & PR & MR & Comments \\
\hline Zelek et al. [36] & Breast $^{\mathrm{a}}$ & 2/3/4 line & $22^{b}$ & - & 4 & 2 & $\begin{array}{l}\text { Two patients had partial response of soft } \\
\text { tissue disease with SD in bone metastases. } \\
\text { Median survival }=10 \text { months }^{c}\end{array}$ \\
\hline Curigliano et al. [37] & Ovarian ${ }^{\mathrm{d}}$ & 2 line & 17 & 1 & 3 & 2 & Activity in liver metastases \\
\hline
\end{tabular}

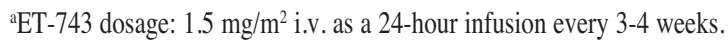

${ }^{\text {b}} 27$ patients entered/22 with measurable disease.

'Intention-to-treat analysis.

'ET-743 dosage: $1.3-1.6 \mathrm{mg} / \mathrm{m}^{2}$ i.v. as a 3-hour infusion every 3-4 weeks.

Abbreviations: ET-743 = ecteinascidin 743; CR = complete response; $\mathrm{PR}=$ partial response; $\mathrm{MR}=$ minor response; $\mathrm{SD}=$ stable disease.

a therapeutic benefit in patients with advanced soft tissue sarcoma resistant to or relapsed subsequent to doxorubicincontaining chemotherapy [33-35], with long-lasting responses (median duration $=17$ months). Also, preliminary evidence of activity in heavily pretreated advanced breast cancer and in patients with advanced ovarian cancer resistant to or relapsed subsequent to platinum complexes and paclitaxel is being generated [36, 37]. The available results of phase II studies are summarized in
Tables 3 and 4 for soft-tissue sarcoma and epithelial breast and ovarian cancer, respectively.

\section{Conclusions}

We have summarized some of the important molecular mechanisms of action of the natural product ET-743, particularly related to its binding to DNA and its effect on DNA repair and transcriptional regulation. We believe that both the effects on transcription and on the DNA repair 
mechanisms require further evaluation. At this stage, it is still unclear why different promoters are differentially affected, although an initial evaluation of the regulatory DNA sequences tends to exclude that effects are related to a specific DNA sequence. Whether the effects of ET-743 on transcription regulation are connected to NER pathways is still not clear. It may be the case that the antitumor activity of ET-743 is defined by its effect on transcriptional regulation, and that optimal biological activity is achieved with intact NER pathways, but it is uncertain whether intact NER is required for activity. In spite of its as yet undetermined mode of action, there is strong biological and biochemical evidence that the mechanism of action of ET-743 is distinct from those previously described for other anticancer drugs. Also, the relatively high cytotoxicity of ET743 against cells in $\mathrm{G}_{1}$ is a unique feature for a DNA binding drug. The peculiar mechanism of action and the observed biological properties of ET-743 in preclinical systems may help to explain why the ongoing clinical investigations are reporting that this compound can be effective against tumors that are relapsed or refractory to the available anticancer drugs [6-9].

\section{ACKNOWLEDGMENTS}

The generous contribution of the Nerina and Mario Mattioli Foundation is gratefully acknowledged.

\section{REFERENCES}

1 Rinehart KL, Holt TG, Fregeau NL et al. Ecteinascidins 729, 743, 745, 759A, 759B, and 770: potent antitumor agents from the Caribbean tunicate Ecteinascidia turbinata. J Org Chem 1990;55:4512-4515.

2 Guan Y, Sakai R, Rinehart KL et al. Molecular and crystal structures of ecteinascidins: potent antitumor compounds from the Caribbean tunicate Ecteinascidia turbinata. J Biomol Struct Dyn 1993;10:793-818.

3 Jimeno JM, Faircloth G, Cameron L et al. Progress in the acquisition of new marine-derived anticancer compounds: development of ecteinascidin-743 (ET-743). Drugs Fut 1996;21:1155-1165.

4 Izbicka E, Lawrence R, Raymond E et al. In vitro antitumor activity of the novel marine agent, ecteinascidin-743 (ET743, NSC-648766) against human tumors explanted from patients. Ann Oncol 1998;9:981-987.

5 Valoti G, Nicoletti MI, Pellegrino A et al. Ecteinascidin-743, a new marine natural product with potent antitumor activity on human ovarian carcinoma xenografts. Clin Cancer Res 1998;4:1977-1983.

6 Delaloge S, Yovine A, Taamma A et al. Ecteinascidin-743: a marine-derived compound in advanced, pretreated sarcoma patients-preliminary evidence of activity. J Clin Oncol 2001;19:1248-1255.

7 Taamma A, Misset JL, Riofrio M et al. Phase I and pharmacokinetic study of ecteinascidin-743, a new marine compound, administered as a 24-hour continuous infusion in patients with solid tumors. J Clin Oncol 2001;19:1256-1265.

8 van Kesteren C, Cvitkovic E, Taamma A et al. Pharmacokinetics and pharmacodynamics of the novel marine-derived anticancer agent ecteinascidin 743 in a phase I dose-finding study. Clin Cancer Res 2000;6:4725-4732.

9 Ryan DP, Supko JG, Eder JP et al. Phase I and pharmacokinetic study of ecteinascidin 743 administered as a 72-hour continuous intravenous infusion in patients with solid malignancies. Clin Cancer Res 2001;7:231-242.

10 Pommier Y, Kohlhagen G, Bailly C et al. DNA sequence- and structure-selective alkylation of guanine $\mathrm{N} 2$ in the DNA minor groove by ecteinascidin 743 , a potent antitumor compound from the Caribbean tunicate Ecteinascidia turbinata. Biochemistry 1996;35:13303-13309.

11 Moore II, Seaman FC, Hurley LH. NMR-based model of an ecteinascidin 743-DNA adduct. J Am Chem Soc 1997;119:54755476.

12 Zewail-Foote M, Hurley LH. Ecteinascidin 743: a minor groove alkylator that bends DNA toward the major groove. J Med Chem 1999;42:2493-2497.

13 Zewail-Foote M, Li VS, Kohn H et al. The inefficiency of incisions of ecteinascidin 743-DNA adducts by the UvrABC nuclease and the unique structural feature of the DNA adducts can be used to explain the repair-dependent toxicities of this antitumor agent. Chem Biol 2001;8:1033-1049.

14 Seaman FC, Hurley LH. Molecular basis for the DNA sequence selectivity of Ecteinascidin 736 and 743: evidence for the dominant role of direct readout via hydrogen bonding. J Am Chem Soc 1998;120:13028-13041.

15 Bonfanti M, La Valle E, Fernandez Sousa Faro JM et al. Effect of ecteinascidin-743 on the interaction between DNA binding proteins and DNA. Anticancer Drug Des 1999;14:179-186.

16 Minuzzo M, Marchini S, Broggini M et al. Interference of transcriptional activation by the anti-neoplastic drug ecteinascidin743. Proc Natl Acad Sci USA 2000;97:6780-6784.

17 Jin S, Gorfajn B, Faircloth G et al. Ecteinascidin 743, a transcription-targeted chemotherapeutic that inhibits MDR1 activation. Proc Natl Acad Sci USA 2000;97:6775-6779.

18 Martinez EJ, Corey EJ, Owa T. Antitumor activity- and gene expression-based profiling of ecteinascidin Et 743 and phthalascidin Pt 650. Chem Biol 2001;146:1-10.

19 Synold TW, Dussault I, Forman BM. The orphan nuclear receptor SXR coordinately regulates drug metabolism and efflux. Nat Med 2001;7:584-590.

20 Takebayashi Y, Pourquier P, Yoshida A et al. Poisoning of human DNA topoisomerase I by ecteinascidin 743, an anticancer drug that selectively alkylates DNA in the minor groove. Proc Natl Acad Sci USA 1999;96:7196-7201. 
21 Martinez EJ, Owa T, Schreiber SL et al. Phthalascidin, a synthetic antitumor agent with potency and mode of action comparable to ecteinascidin 743. Proc Natl Acad Sci USA 1999;96:3496-3501.

22 Damia G, Silvestri S, Carrassa L et al. Unique pattern of ET-743 activity in different cellular systems with defined deficiencies in DNA repair pathways. Int J Cancer 2001;92:583-588.

23 Takebayashi Y, Goldwasser F, Urasaki Y et al. Ecteinascidin 743 induces protein-linked DNA breaks in human colon carcinoma HCT116 cells and is cytotoxic independently of topoisomerase I expression. Clin Cancer Res 2001;7:185-191.

24 Erba E, Bergamaschi D, Bassano L et al. Ecteinascidin-743 (ET-743), a natural marine compound, with a unique mechanism of action. Eur J Cancer 2001;37:97-105.

25 Takebayashi Y, Pourquier P, Zimonjic DB et al. Antiproliferative activity of ecteinascidin 743 is dependent upon transcription-coupled nucleotide-excision repair. Nat Med 2001;7:961-966.

26 Li WW, Takahashi N, Jhanwar S et al. Sensitivity of soft tissue sarcoma cell lines to chemotherapeutic agents: identification of ecteinascidin-743 as a potent cytotoxic agent. Clin Cancer Res 2001;7:2908-2911.

27 Vikhanskaya F, Marchini S, Marabese M et al. p73 $\alpha$ overexpression is associated with resistance to treatment with DNAdamaging agents in a human ovarian cancer cell line. Cancer Res 2001;61:935-938.

28 Erba E, Muradore I, Tiozzo G et al. ET-743 and cisplatin (DDP) show in vitro and in vivo synergy against human sarcoma and ovarian carcinoma cell lines. Proceedings of the AACR-NCI-EORTC International Conference on Molecular Targets and Cancer Therapeutics: Discovery, Biology and Clinical Applications. Miami Beach, Florida, 2001:83a.

29 Takahashi N, Li WW, Banerjee D et al. Sequence-dependent enhancement of cytotoxicity produced by ecteinascidin 743 (ET-743) with doxorubicin or paclitaxel in soft tissue sarcoma cells. Clin Cancer Res 2001;7:3251-3257.

30 Villalona-Calero MA, Eckhardt SG, Weiss G et al. A phase I and pharmacokinetic study of ecteinascidin-743 on a daily $\times$
5 schedule in patients with solid malignancies. Clin Cancer Res 2002;8:75-85.

31 Twelves C, Hoekman H, Bowman A et al. A phase I and pharmacokinetic (PK) study of ET-743 evaluating a 3 hours (h) intravenous (iv) infusion (I) in patients (pts) with solid tumors. Proceedings of the AACR-NCI-EORTC International Conference on Molecular Targets and Cancer Therapeutics. Washington, DC, 1999:307a.

32 Forouzesh B, Hidalgo M, Denis L et al. Phase I and pharmacokinetic study of ET-743, a minor groove DNA binder, administrated weekly to patients with advanced cancer. Proc Am Soc Clin Oncol 2001;20:94a.

33 Yovine A, Riofrio M, Brain E et al. Ecteinascidin-743 given as a 24 hour $(\mathrm{H})$ intravenous continuous infusion (IVCI) every 3 weeks: results of a Phase II trial in patients (PTS) with pretreated soft tissue sarcomas (PSTS). Proc Am Soc Clin Oncol 2001;20:363a.

34 Demetri G, Manola J, Harmon D et al. Ecteinascidin-743 (ET-743) induces durable responses and promising 1-year survival rates in soft tissue sarcomas (STS): final results of phase II and pharmacokinetic studies in the U.S.A. Proc Am Soc Clin Oncol 2001;20:352a.

35 Le Cesne A, Blay J, Judson I et al. ET-743 is an active drug in adult soft-tissue sarcoma: a STBSG-EORTC Phase II trial. Proc Am Soc Clin Oncol 2001;20:353a.

36 Zelek L, Yovine A, Brain E et al. Preliminary results of phase II study of ecteinascidin-743 (ET-743) with the 24 hour $(\mathrm{H})$ continuous infusion $(\mathrm{CI})$ Q3weeks schedule in pretreated advanced/metastatic breast cancer (A/MBC) patients (Pts). Proceedings of the 11th NCI-EORTC-AACR Symposium on New Drugs in Cancer Therapy. Amsterdam, The Netherlands, 2000:85a.

37 Curigliano G, Bauer J, Capri G et al. Ecteinascidin 743 (ET743 ) in ovarian cancer: activity in xenografts and preliminary results of an ongoing study in patients failing platinum-taxanes. Proceedings of the AACR-NCI-EORTC International Conference on Molecular Targets and Cancer Therapeutics: Discovery, Biology and Clinical Applications. Miami Beach, Florida, 2001:78a. 